\title{
Gene expression profile analysis of pancreatic cancer based on microarray data
}

\author{
JIN LONG, ZHE LIU, XINGDA WU, YUANHONG XU and CHUNLIN GE \\ Department of General Surgery, The First Hospital of China Medical University, Shenyang, Liaoning 110001, P.R. China
}

Received April 9, 2015; Accepted March 4, 2016

DOI: $10.3892 / \mathrm{mmr} .2016 .5021$

\begin{abstract}
The present study identified differentially-expressed genes (DEGs) between pancreatic cancer (PC) tissues and normal tissues, and assessed genetic factors associated with the pathogenesis of PC. The mRNA expression microarray dataset, GSE16515, containing 52 samples, including 16 paired tumor and normal tissue samples, and 20 tumor samples, was downloaded from Gene Expression Omnibus. Raw data were normalized and DEGs were identified. Subsequently, clustering was performed, protein-protein interaction networks were drawn, and functional and pathway enrichment analyses of the DEGs were performed. Copy number variations of DEGs were also identified. A total of 1,765 DEGs between PC and normal tissues were identified, including 1,312 upregulated and 453 downregulated DEGs. Upregulated DEGs were associated with the regulation of nucleocytoplasmic and intracellular transport, whereas downregulated DEGs were associated with the response to organic substances and hormone stimulus. The pancreatic cancer pathway was connected to three DEGs, namely transforming growth factor $\beta 1$ (TGFB1), TGF $\beta$ receptor $1(T G F B R 1)$ and epidermal growth factor $(E G F)$, which had 2, 3 and $5 \mathrm{CNVs}$, respectively. These results indicated the important roles of TGFBl, TGFBRI and EGF in the pathogenesis of PC. These genes may be potential therapeutic targets for the treatment of PC.
\end{abstract}

\section{Introduction}

Pancreatic cancer (PC; OMIM 260350) is a highly lethal disease, with an incidence rate that is constantly increasing (1). The 5-year survival rate of $\mathrm{PC}$ is $<5 \%$ (2), and almost all patients with primary PC develop metastases.

Previous studies have indicated that PC has a complex genomic landscape with frequent copy number variations (CNVs)

Correspondence to: Dr Jin Long, Department of General Surgery, The First Hospital of China Medical University, 155 Nanjing Bei Street, Shenyang, Liaoning 110001, P.R. China

E-mail: jinlongjllj@163.com

Key words: pancreatic cancer, differentially-expressed genes, $T G F B 1, T G F B R 1, E G F$ or copy number polymorphisms (CNPs) (3). Biankin et al (4) defined 16 significantly mutated genes [e.g., Kirsten rat sarcoma viral oncogene homolog (KRAS), tumor protein p53 (TP53), SMAD family member $4(S M A D 4)$ and transforming growth factor $\beta$ receptor 2 (TGFBR2)] that were reaffirmed known mutations associated with PC. The commonly mutated genes, such as KRAS (74-100\%), cyclin-dependent kinase inhibitor 2A (up to $98 \%$ ), TP53 (43-76\%), erb-b2 receptor tyrosine kinase 2 (ERBB2; $\sim 65 \%)$ and fragile histidine triad ( 70\%) have been found in PC (5-9). Among these genes, KRAS and ERBB2 are proto-oncogenes, whereas the other genes are tumor suppressors (3). The progression of PC is correlated with the activation of oncogenes and the inactivation of tumor suppressor genes, as well as the deregulation of a number of signaling pathways, among which the epidermal growth factor receptor (EGFR), v-akt murine thymoma viral oncogene homolog $1(v-A K T 1)$ and nuclear factor of $\kappa$ light polypeptide gene enhancer in B-cells 1 $(N F \kappa B 1)$ pathways appear to be most relevant (10).

$A K T 1$ is a central regulator of cell growth. AKT1 has been shown to inhibit apoptosis and promote cell survival, thus contributing to the pathogenesis of cancer $(11,12)$. Pei et al $(13)$ showed that FK506-binding protein 51 (FKBP51) acted as a scaffolding protein for Akt and that it promoted the activation of Akt. The expression of FKBP51 was downregulated in PC tissues. Decreased FKBP51 expression resulted in the hyperphosphorylation of Akt, and then decreased the level of cell death in the PC tissues. Thus, Pei et al demonstrated FKBP51 to be a negative regulator of the Akt pathway (13). Pei et al also released the mRNA expression microarray dataset, GSE16515, consisting of 36 pancreatic tumor and 16 normal tissue samples. Numerous studies have since been performed using this dataset (14-16). For example, using the GSE16515 dataset, Yang et al (14) screened the differentially-expressed genes (DEGs), such as TGF $\alpha$ (TGFA) and EGF, between PC tumor tissues and normal tissues, and selected the important single nucleotide polymorphisms (SNPs) of A/G and C/T in the DEGs. However, none of the studies based on the GSE16515 dataset performed an analysis of CNVs in the DEGs.

Using the GSE16515 dataset downloaded from Gene Expression Omnibus (GEO), the DEGs between PC tumor tissues and normal tissues were screened in the present study. Next, clustering analysis and construction of a protein-protein interaction (PPI) network of DEGs was performed. The underlying functions of these DEGs were investigated by functional and pathway enrichment analyses. Finally, the CNVs of these 
DEGs were also analyzed. This will be beneficial for developing therapeutic strategies for patients with PC.

\section{Materials and methods}

mRNA microarray data. The mRNA expression microarray data from the GSE16515 dataset (6) was downloaded from GEO (http://www.ncbi.nlm.nih.gov/geo/), based on the platform of GPL570 [HG-U133_Plus_2] Affymetrix Human Genome U133 Plus 2.0 Array (Affymetrix, Santa Clara, CA, USA). GSE16515 was composed of 52 samples (from 34 males and 18 females). In total, 32 samples consisted of tumor and normal expression data, whereas 20 samples consisted of only tumor data. These samples were obtained during clinically indicated surgical procedures and consent was obtained for experimental purposes. The raw data and the probe annotation files were downloaded for further analysis. The microarray data of the GSE16515 dataset was analyzed following the procedures presented in Fig. 1.

Data preprocessing and DEG identification. The Robust Multiarray Average in Affy package of R (http://www.bioconductor.org/packages/release/bioc/html/affy.html), provided by Bioconductor project (17), was applied to process the raw microarray data. The processing included background correction, quantile normalization and probe summarization of expression values. The gene expression matrices were obtained for further analysis. Afterwards, the Linear Models for Microarray Data package was used to identify the gene signatures between the tumor and normal tissues, with significant differences indicated using a P-value of $<0.05$. Next, the Bonferroni correction (18) was applied to adjust the raw P-value for the false discovery rate (FDR) and to calculate the fold change (FC). In the present study, the cut-off criteria for the statistically significant DEGs were $\mid \log 2 \mathrm{FCl}>1$ and $\mathrm{FDR}<0.05$.

Clustering analysis of DEGs. Based on the Euclidean distance between the expression profile of each DEG filtered from the samples, hierarchical clustering can be used to build a hierarchy of clusters of DEGs (19). The heatmap figure of the DEGs was drawn with the R package pheatmap (http://cran.r-project.org/web/packages/pheatmap/index.html) function. DEGs with the same signatures were clustered together, indicating the specificity of the DEGs.

Identification of PPIs of DEGs. Identification of protein complexes and functional modules from PPI networks is crucial to predict protein functions and to understand the principles of cellular organization (20). The Search Tool for the Retrieval of Interacting Genes (STRING; http://string-db.org/) database provides uniquely comprehensive coverage and ease of access for the prediction of interaction information (21). To better understand the interactions of the DEGs, the PPI network of their encoding products was predicted using the STRING database, with the reliability threshold of $>0.9$. Cytoscape software (http://cytoscape.org/), a standard tool for the integrated analysis and visualization of biological networks, was used to visualize the PPI network (22).

Functional enrichment analysis of DEGs. Gene Ontology (GO; http://www.geneontology.org/) analysis is an functional study

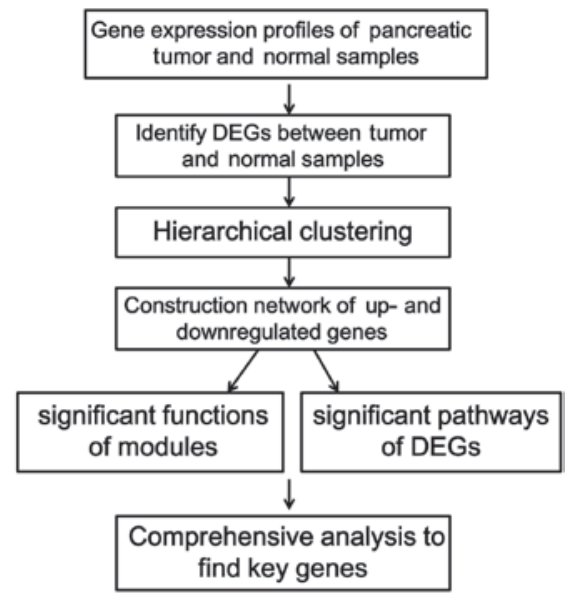

Figure 1. Flowchart of the analysis of the microarray data from the GSE16515 dataset. DEG, differentially-expressed gene.

method for large-scale transcriptomic or genomic data (23). In order to investigate the biofunctions of DEGs in tumor progression, the Database for Annotation, Visualization and Integrated Discovery (http://david.abcc.Ncifcrf.gov/), a high-throughput and integrated data-mining environment (24), was used to identify the enriched GO biological processes that the DEGs were associated with $(\mathrm{FDR}<0.05)$.

Pathwayanalysis ofDEGs. The KyotoEncyclopedia of Genes and Genomes (KEGG; http://www.genome.jp/kegg/pathway.html) pathway database provides information on how molecules or genes function (25). Pathway analysis of all the DEGs was performed using the KEGG database. The KEGG maps of biological functions associated with DEGs were obtained $(\mathrm{P}<0.05)$.

CNV analysis of DEGs. The Database of Genomic Variants (DGV; http://dgv.tcag.ca/) (26) was used to identify the CNVs in the DEGs, including deletions, insertions, duplications, complex multi-site variants and SNPs.

\section{Results}

Data processing and identification of DEGs. After the normalization, the DEGs between the tumor and normal tissues of the 52 samples were identified, with the cut-off criteria of $\mid \log 2 \mathrm{FCl}>1$ and FDR $<0.05$. A total of 1,765 DEGs were identified between the PC and normal tissues, of which 1,312 were upregulated and 453 were downregulated.

Hierarchical clustering of DEGs. Hierarchical clustering of the 1,765 DEGs is presented in Fig. 2. The LogFC values of the DEGs ranged from 6-fold downregulated and 6-fold upregulated. The majority of the DEGs were upregulated in the PC tumors compared with the normal tissues. The tumor samples and the normal control samples could easily be distinguished from the characteristics of the DEGs.

PPIs analysis of DEGs. To identify the PPIs and predict protein functions, PPI network analysis was performed using the STRING database (threshold $>0.9$ ). The resulting PPI network 
Table I. Enriched GO biological processes of the DEGs in the protein-protein interaction networks.

\begin{tabular}{|c|c|c|c|c|}
\hline Term and function & Count & Genes & P-value & FDR \\
\hline \multicolumn{5}{|l|}{ Upregulated DEGs } \\
\hline $\begin{array}{l}\text { GO:0046822 - Regulation of } \\
\text { nucleocytoplasmic transport }\end{array}$ & 7 & $\begin{array}{l}C D K N 2 A, T G F B R 1, S M A D 3, C D H 1, \\
T A C C 3, F L N A, T G F B 1\end{array}$ & $1.72 \times 10^{-6}$ & 0.002848 \\
\hline $\begin{array}{l}\text { GO: } 0032880 \text { - Regulation of } \\
\text { protein localization }\end{array}$ & 9 & $\begin{array}{l}\text { TGFBR1, SMAD3, CDH1, CDH2, } \\
\text { CASP1, TACC3, FLNA, TGFB1, IL1A }\end{array}$ & $2.60 \times 10^{-6}$ & 0.004308 \\
\hline $\begin{array}{l}\text { GO:0051222 - Positive regulation } \\
\text { of protein transport }\end{array}$ & 7 & $\begin{array}{l}\text { TGFBR1, SMAD3, CDH1, CASP1, } \\
\text { FLNA, TGFB1, IL1A }\end{array}$ & $4.05 \times 10^{-6}$ & 0.006710 \\
\hline $\begin{array}{l}\text { GO:0032386 - Regulation of } \\
\text { intracellular transport }\end{array}$ & 7 & $\begin{array}{l}C D K N 2 A, T G F B R 1, S M A D 3, C D H 1, \\
T A C C 3, F L N A, T G F B 1\end{array}$ & $5.69 \times 10^{-6}$ & 0.009436 \\
\hline \multicolumn{5}{|l|}{ Downregulated DEGs } \\
\hline $\begin{array}{l}\text { GO:0043085 - Positive } \\
\text { regulation of catalytic activity }\end{array}$ & 13 & $\begin{array}{l}\text { ADCY1, PTGER3, CCKBR, C6, C5, } \\
\text { LPAR3, EDNRB, PRKAR2B, PLCE1, } \\
\text { CLPS, GNAS, EGF, PSMD 6 }\end{array}$ & $1.72 \times 10^{-5}$ & 0.027131 \\
\hline $\begin{array}{l}\text { GO:0010033 - Response to } \\
\text { organic substances }\end{array}$ & 15 & $\begin{array}{l}\text { TF, ADCY1, PNLIPRP1, GATM, } \\
\text { PDE } 3 B, E P H X 1, C F T R, P D E 3 A, \\
\text { NPY1R, PRKAR } 2 B, A B A T, A N G P T 1, \\
\text { GNAS, SST, GNG7 }\end{array}$ & $2.20 \times 10^{-5}$ & 0.034801 \\
\hline $\begin{array}{l}\text { GO:0009725 - Response to } \\
\text { hormone stimuli }\end{array}$ & 11 & $\begin{array}{l}\text { PRKAR2B, PNLIPRP1, ADCY1, GATM, } \\
\text { PDE } 3 B, G N A S, A N G P T 1, C F T R, \\
\text { NPY1R, SST, GNG7 }\end{array}$ & $2.29 \times 10^{-5}$ & 0.036206 \\
\hline $\begin{array}{l}\text { GO:0006575 - Cellular amino } \\
\text { acid derivative metabolic processes }\end{array}$ & 8 & $\begin{array}{l}\text { GSTA1, P4HB, CTH, GATM, GPX3, } \\
A B A T, G A M T, G N M T\end{array}$ & $2.78 \times 10^{-5}$ & 0.043955 \\
\hline
\end{tabular}

FDR, false discovery rate; DEG, differentially-expressed genes; GO, gene ontology.

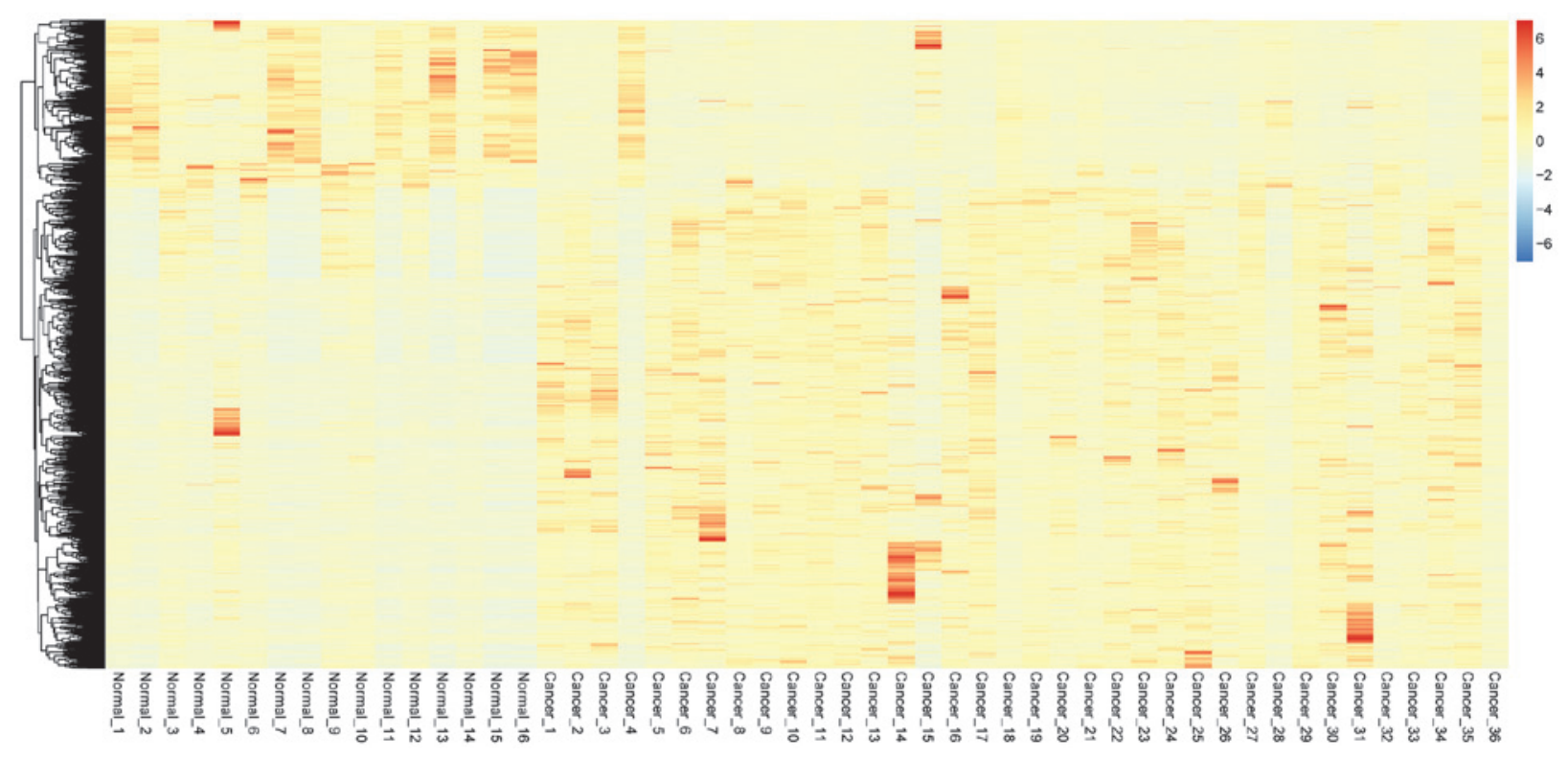

Figure 2. Heat map clustering of the differentially-expressed genes between two samples. The x-axis represents normal and tumor samples, and the y-axis represents genes. Blue $(<0)$ indicates downregulation and orange $(>0)$ indicates upregulation of gene expression in the pancreatic and normal tissues.

of upregulated DEGs connected to 92 nodes (proteins) through 171 PPIs, whereas the PPI network of the downregulated DEGs connected to 82 nodes through 83 PPIs (Fig 3).

Functional enrichment analysis of DEGs. To obtain the enriched GO biological processes of the DEGs in the PPI networks, GO functional enrichment analysis was performed for the up- and downregulated DEGs, respectively (FDR <0.05). The upregulated DEGs (including TGFBl and TGFBRI) were associated with significant biological processes, such as the regulation of nucleocytoplasmic transport, protein localization and intracellular transport (Table I), whereas the downregulated DEGs 


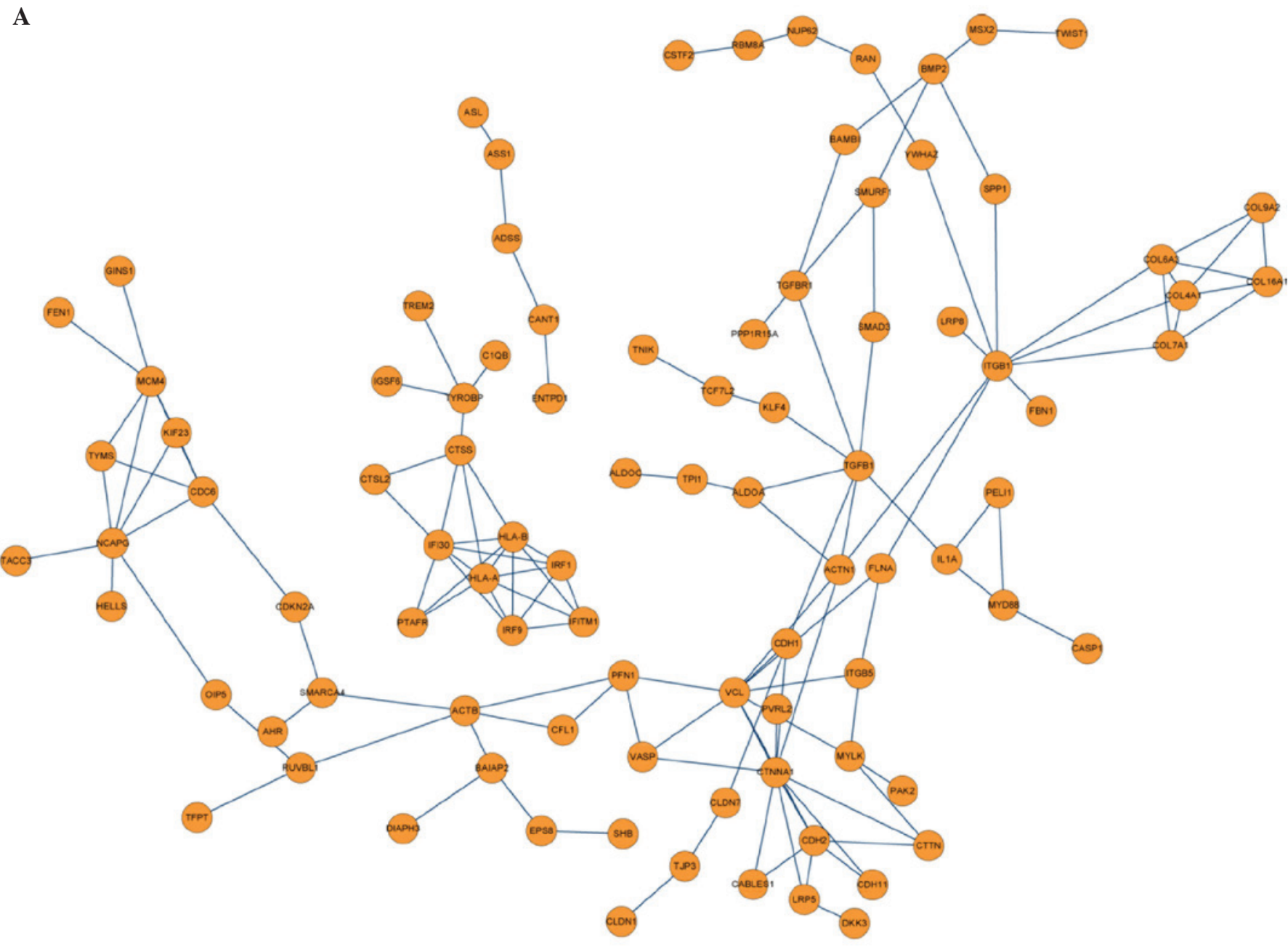

B

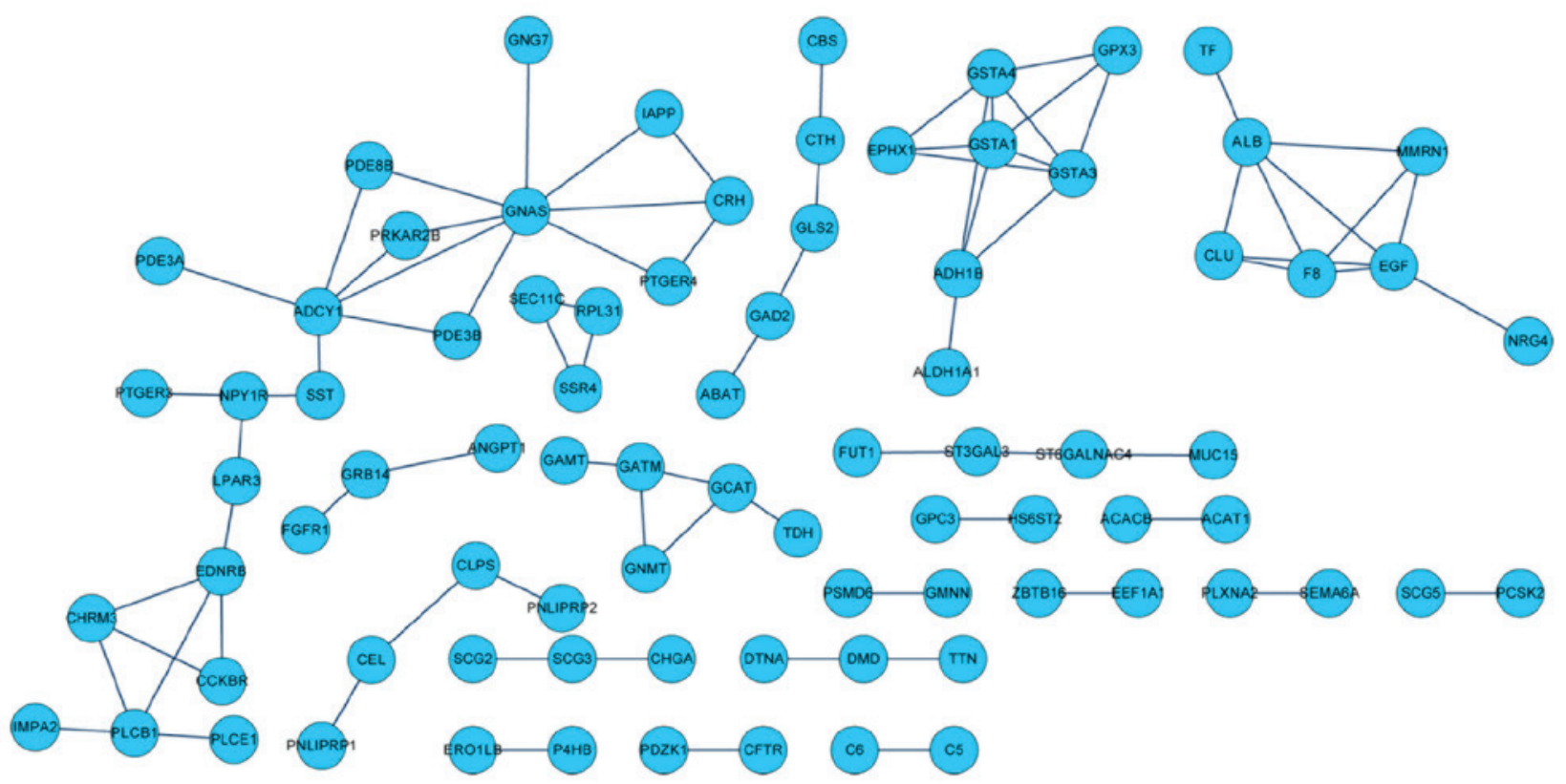

Figure 3. Protein-protein interaction networks of (A) upregulated DEGs (orange) and (B) downregulated DEGs (blue). The nodes represent proteins and the lines between nodes represent interactions. DEG, differentially-expressed gene.

(e.g., $E G F$ ) were correlated with the biological processes of the positive regulation of catalytic activity, the response to organic substances and the response to hormone stimuli (Table I).
Pathway analysis of DEGs. The KEGG maps of biological functions associated with DEGs in the PPI networks were obtained $(\mathrm{P}<0.05)$. The results showed that only the pancreatic 
Table II. Copy number variations in TGFB1, TGFBRl and EGF genes.

\begin{tabular}{|c|c|c|c|c|}
\hline First author, year & Gene & Variant ID & Subtype & (Ref.) \\
\hline Xu et al, 2011 & $T G F B 1$ & nsv911769 & Loss & (27) \\
\hline Shaikh et al, 2009 & & nsv521311 & Insertion & (28) \\
\hline $\mathrm{Xu}$ et al, 2011 & $T G F B R 1$ & $\begin{array}{l}\text { nsv893619 } \\
\text { nsv893618 }\end{array}$ & $\begin{array}{l}\text { Insertion } \\
\text { Insertion }\end{array}$ & (27) \\
\hline Wong et al, 2007 & & nsv831666 & Insertion & (29) \\
\hline Abecasis et al, 2012 & $E G F$ & esv2672203 & Deletion & (30) \\
\hline McKernan et al, 2009 & & esv2618042 & Insertion & (31) \\
\hline Conrad et al, 2010 & & esv22936 & Loss & (32) \\
\hline Mills et al, 2006 & & nsv290769 & Loss & (33) \\
\hline Kim et al, 2009 & & nsv820232 & Loss & (34) \\
\hline
\end{tabular}

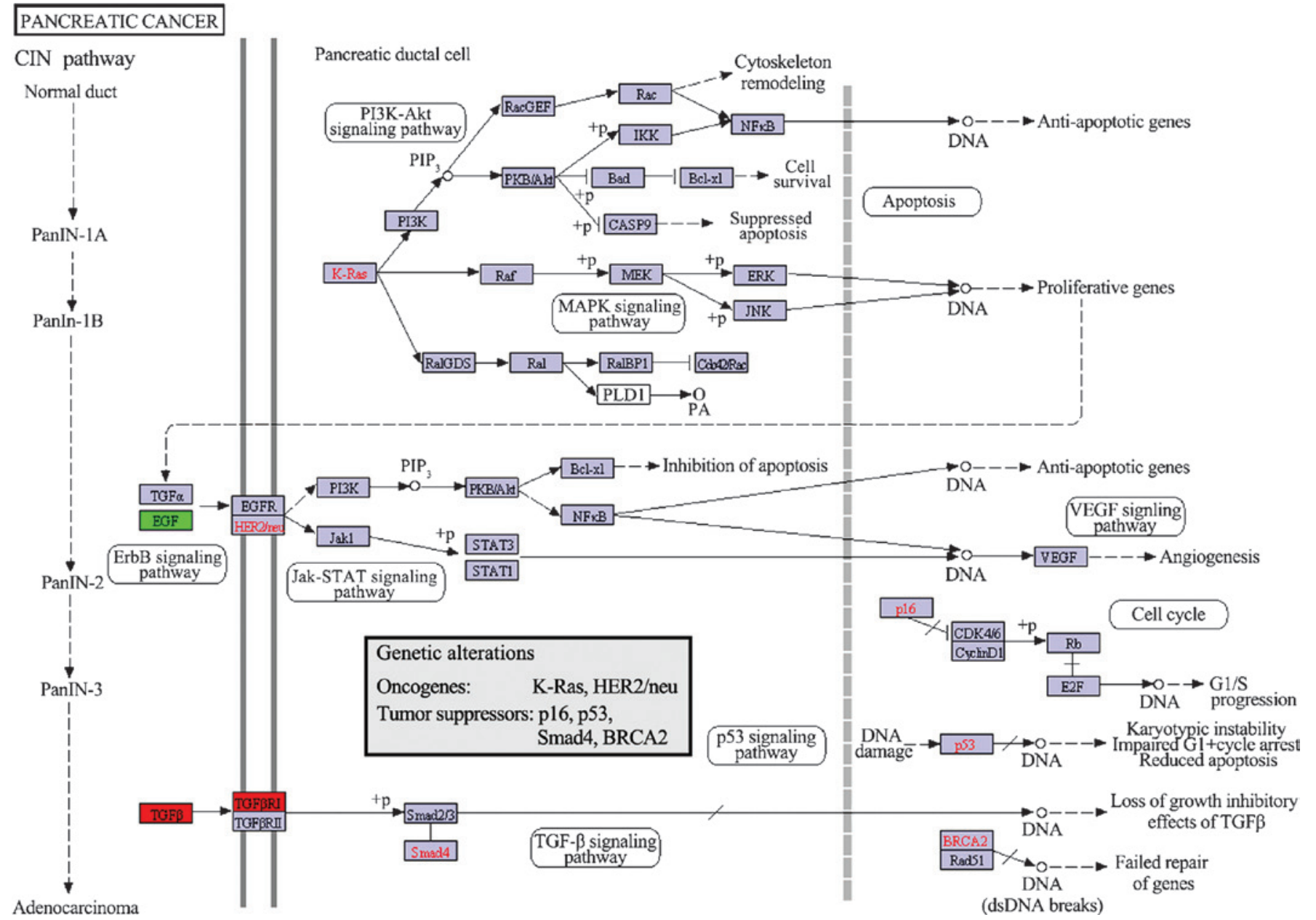

Figure 4. Molecular pathways in pancreatic cancer involving the DEGs in the protein-protein interaction networks. Red boxes represent upregulated DEGs and the green box represents a downregulated DEG. Red letters represent tumor suppressors or oncogenes that have been validated in previous studies. CIN, chromosomal instability; DEG, differentially-expressed gene; ds, double strand; PanIN, pancreatic intraepithelial neoplasia.

cancer pathway was associated with DEGs in the PPI networks, including upregulated TGFB1 and TGFBR1, and downregulated $E G F$ (Fig. 4).

CNV analysis of DEGs. The CNVs of the TGFB1, TGFBRI and $E G F$ genes were further identified using the DGV. The identification of CNVs of DEGs included deletions, insertions, duplications and complex multi-site variants. Finally, 2, 3 and 5 CNVs, were identified in the TGFB1, TGFBR1 and $E G F$ genes, respectively (Table II) (27-34). In total, 1 of the CNVs of TGFB1 was insertin; all 3 of the CNVs of TGFBRI were insertins; and 1 of the $5 \mathrm{CNVs}$ of $E G F$ was insertin. 


\section{Discussion}

The early stages of PC are usually asymptomatic, and the majority of patients with PC are diagnosed at an advanced stage. The pathogenesis of PC is involved in a number of biological processes. Advanced studies of genetic factors have greatly improved our understanding of the pathogenesis of PC, which is associated with gene mutations, continuous changes to nuclei, loss of polarity and changes in cellular architecture (35).

In the present study, the DEGs between PC tumor tissues and normal tissues were systematically investigated. A total of 1,765 DEGs, including 1,312 upregulated and 453 downregulated DEGs, were identified. The majority of DEGs were upregulated in the tumor tissues. The upregulated DEGs (including $S M A D 3, T G F B 1$ and $T G F B R 1$ ) were associated with the regulation of nucleocytoplasmic and intracellular transport, and protein localization, whereas the downregulated DEGs (e.g., $E G F$ ) were associated with regulation of catalytic activity, and the responses to organic substances and hormone stimuli. All 4 of these DEGs were connected to the pancreatic cancer pathway. In addition, TGFB1, TGFBR1 and EGF exhibited 2, 3 and $5 \mathrm{CNVs}$, respectively. These results proposed an important role for these DEGs in PC development.

CNVs or CNPs, such as deletions, insertions, duplications and complex multi-site variants, have been found in all humans and in other mammals (36). CNVs can be simple in structures like tandem duplication, or may be complex in the genome, such as in gains or losses of homologous sequences at multiple sites (37). Yang et al (14) screened the DEGs between PC tissues and normal tissues using the GSE16515 dataset, and selected the important SNPs of A/G and C/T in DEGs such as $T G F A$ and $E G F$. In the present study, $5 \mathrm{CNVs}$ of $E G F$ were identified, including 3 losses, 1 deletion and 1 insertion. These results demonstrate that the CNVs of $E G F$ may be significant in the pathogenesis of PC.

$E G F R$ is required for $K R A S$-induced PC (38). Accordingly, the overexpression of EGFR and TGFA has been reported as an important molecular abnormality in human PCs (39). EGF and TGFA are essential molecules for the VEGF signaling pathway (Fig. 4). The inhibition of the vascular endothelial growth factor (VEGF) signaling pathway is beneficial for the suppression of tumor metastasis and invasion, such as required in PC (40). In the present study, it was found that the expression of $E G F$ was downregulated in the PC tissues. Taken together, these results suggest the significant role of $E G F$ in $\mathrm{PC}$ and indicate that $E G F$ may be a novel target for the therapy of PC.

$T G F B 1$ and TGFBR1 were overexpressed in the PC tumors in the present study. The two genes are elements of the TGFB signaling pathway, which is a potent inhibitor of cell growth (41). There is growing evidence that members of the $T G F B$ family are frequently mutated in cancer. CNVs, DNA segments that are $\geq 1 \mathrm{~kb}$ and present at variable copy number in comparison with a reference genome, affect the expression of genes, the variation and adaptation of phenotypes, and the pathogenesis of diseases, such as human immunodeficiency virus-1 infection, by disrupting genes and altering gene dosage in microdeletion or microduplication disorders $(42,43)$. The present study identified 3 insertion CNVs in TGFBRI, and 1 insertion CNV and 1 loss CNV in TGFB1. Moreover, 3 of the CNVs in TGFBRI and TGFBI were located at the same segment as in a previous study (27).These indicate the vital roles of TGFBRI and TGFB1 in PC development. The genes may be potential therapeutic targets for the treatment of PC.

In summary, 1,765 DEGs, including 1,312 upregulated (e.g., $T G F B 1$ and $T G F B R I$ ) and 453 downregulated (e.g., $E G F)$ DEGS, were identified in the PC tissues compared with the normal tissues in the present study. The upregulated DEGs were associated with the regulation of nucleocytoplasmic and intracellular transport, whereas the downregulated DEGs were associated with the regulation of catalytic activity, and the response to organic substances and hormone stimuli. A pancreatic cancer pathway was connected to the DEGs of $T G F B 1, T G F B R 1$ and $E G F$. In addition, TGFB1, TGFBRI and $E G F$ exhibited 2, 3 and $5 \mathrm{CNVs}$, respectively. These results suggested the significance of the DEGs in PC. TGFB1, $T G F B R 1$ and $E G F$ may be potential therapeutic targets for the treatment of PC. However, further clinical trials are required to validate these conclusions and hypotheses.

\section{References}

1. Lowenfels $\mathrm{AB}$ and Maisonneuve P: Epidemiology and risk factors for pancreatic cancer. Best Pract Res Clin Gastroenterol 20: 197-209, 2006.

2. Thomas JK, Kim MS, Balakrishnan L, Nanjappa V, Raju R, Marimuthu A, Radhakrishnan A, Muthusamy B, Khan AA, Sakamuri S, et al: Pancreatic Cancer Database: An integrative resource for pancreatic cancer. Cancer Biol Ther 15: 963-967, 2014.

3. Strimpakos A, Saif MW and Syrigos KN: Pancreatic cancer: From molecular pathogenesis to targeted therapy. Cancer Metastasis Rev 27: 495-522, 2008.

4. Biankin AV, Waddell N, Kassahn KS, Gingras MC, Muthuswamy LB, Johns AL, Miller DK, Wilson PJ, Patch AM, Wu J, et al: Pancreatic cancer genomes reveal aberrations in axon guidance pathway genes. Nature 491: 399-405, 2012.

5. Hingorani SR, Petricoin EF, Maitra A, Rajapakse V, King C, Jacobetz MA, Ross S, Conrads TP, Veenstra TD, Hitt BA, et al: Preinvasive and invasive ductal pancreatic cancer and its early detection in the mouse. Cancer Cell 4: 437-450, 2003.

6. Goldstein AM, Fraser MC, Struewing JP, Hussussian CJ, Ranade K, Zametkin DP, Fontaine LS, Organic SM, Dracopoli NC and Clark WH Jr: Increased risk of pancreatic cancer in melanoma-prone kindreds with p16INK4 mutations. N Engl J Med 333: 970-974, 1995

7. Pellegata NS, Sessa F, Renault B, Bonato M, Leone BE, Solcia E and Ranzani GN: K-ras and p53 gene mutations in pancreatic cancer: Ductal and nonductal tumors progress through different genetic lesions. Cancer Res 54: 1556-1560, 1994.

8. Grau AM, Zhang L, Wang W, Ruan S, Evans DB, Abbruzzese JL, Zhang W and Chiao PJ: Induction of p21waf1 expression and growth inhibition by transforming growth factor beta involve the tumor suppressor gene DPC4 in human pancreatic adenocarcinoma cells. Cancer Res 57: 3929-3934, 1997.

9. Sorio C, Baron A, Orlandini S, Zamboni G, Pederzoli P, Huebner $\mathrm{K}$ and Scarpa A: The FHIT gene is expressed in pancreatic ductular cells and is altered in pancreatic cancers. Cancer Res 59: 1308-1314, 1999.

10. Sarkar FH, Banerjee S and Li Y: Pancreatic cancer: Pathogenesis, prevention and treatment. Toxicol Appl Pharmacol 224: 326-336, 2007.

11. Luo J, Manning BD and Cantley LC: Targeting the PI3K-Akt pathway in human cancer: Rationale and promise. Cancer Cell 4: 257-262, 2003.

12. Manning BD and Cantley LC: AKT/PKB signaling: Navigating downstream. Cell 129: 1261-1274, 2007.

13. Pei H, Li L, Fridley BL, Jenkins GD, Kalari KR, Lingle W, Petersen G, Lou Z and Wang L: FKBP51 affects cancer cell response to chemotherapy by negatively regulating Akt. Cancer Cell 16: 259-266, 2009.

14. Yang D, Zhu Z, Wang W, Shen P, Wei Z, Wang C and Cai Q: Expression profiles analysis of pancreatic cancer. Eur Rev Med Pharmacol Sci 17: 311-317, 2013. 
15. Zhao LL, Zhang T, Zhuang LW, Yan BZ, Wang RF and Liu BR Uncovering the pathogenesis and identifying novel targets of pancreatic cancer using bioinformatics approach. Mol Biol Rep 41: 4697-4704, 2014

16. Söderquist F, Hellström PM and Cunningham JL: Human gastroenteropancreatic expression of melatonin and its receptors MT1 and MT2. PLoS One 10: e120195, 2015.

17. Irizarry RA, Hobbs B, Collin F, Beazer-Barclay YD, Antonellis KJ, Scherf U and Speed TP: Exploration, normalization and summaries of high density oligonucleotide array probe level data. Biostatistics 4: 249-264, 2003.

18. Benjamini Y and Hochberg Y: Controlling the false discovery rate: a practical and powerful approach to multiple testing. J R Stat Soc B 57: 289-300, 1995.

19. Deza MM and Deza E: Encyclopedia of distances. Springer, Heidelberg, 2009.

20. Li M, Wu X, Wang J and Pan Y: Towards the identification of protein complexes and functional modules by integrating PPI network and gene expression data. BMC Bioinformatics 13: 109, 2012.

21. Szklarczyk D, Franceschini A, Kuhn M, Simonovic M, Roth A, Minguez P, Doerks T, Stark M, Muller J, Bork P, et al: The STRING database in 2011: Functional interaction networks of proteins, globally integrated and scored. Nucleic Acids Res 39 (Database issue): D561-D568, 2011.

22. Shannon P, Markiel A, Ozier O, Baliga NS, Wang JT, Ramage D, Amin N, Schwikowski B and Ideker T: Cytoscape: A software environment for integrated models of biomolecular interaction networks. Genome Res 13: 2498-2504, 2003.

23. Hulsegge I, Kommadath A and Smits MA: Globaltest and GOEAST: Two different approaches for Gene Ontology analysis. BMC Proc 3 (Suppl 4): S10, 2009.

24. Huang da W, Sherman BT and Lempicki RA: Systematic and integrative analysis of large gene lists using DAVID bioinformatics resources. Nat Protoc 4: 44-57, 2009.

25. Kanehisa M and Goto S: KEGG: Kyoto encyclopedia of genes and genomes. Nucleic Acids Res 28: 27-30, 2000.

26. MacDonald JR, Ziman R, Yuen RK, Feuk L and Scherer SW: The Database of Genomic Variants: A curated collection of structural variation in the human genome. Nucleic Acids Res 42 (Database issue): D986-D992, 2014.

27. Xu H, Poh WT, Sim X, Ong RT, Suo C, Tay WT, Khor CC, Seielstad M, Liu J, Aung T, et al: SgD-CNV, a database for common and rare copy number variants in three Asian populations. Hum Mutat 32: 1341-1349, 2011

28. Shaikh TH, Gai X, Perin JC, Glessner JT, Xie H, Murphy K, O'Hara R, Casalunovo T, Conlin LK, D'Arcy M, et al: High-resolution mapping and analysis of copy number variations in the human genome: a data resource for clinical and research applications. Genome Res 19: 1682-1690, 2009.

29. Wong KK, deLeeuw RJ, Dosanjh NS, Kimm LR, Cheng Z, Horsman DE, MacAulay C, Ng RT, Brown CJ, Eichler EE and Lam WL A comprehensive analysis of common copy-number variations in the human genome. Am J Hum Genet 80: 91-104, 2007.
30. Abecasis GR, Auton A, Brooks LD, Durbin RM, Garrison EP, Kang HM, Korbel JO, Marchini JL, McCarthy S, McVean GA, Abecasis GR; 1000 Genomes Project Consortium: An integrated map of genetic variation from 1,092 human genomes. Nature 491: 56-65, 2012.

31. McKernan KJ, Peckham HE, Costa GL, McLaughlin SF, Fu Y, Tsung EF, Clouser CR, Duncan C, Ichikawa JK, Lee $C C$, et al: Sequence and structural variation in a human genome uncovered by short-read, massively parallel ligation sequencing using two-base encoding. Genome Res 19 $1527-1541,2009$.

32. Conrad DF, Pinto D, Redon R, Feuk L, Gokcumen O, Zhang Y, Aerts J, Andrews TD, Barnes C, Campbell P, et al: Origins and functional impact of copy number variation in the human genome. Nature 464: 704-712, 2010

33. Mills RE, Luttig CT, Larkins CE, Beauchamp A, Tsui C, Pittard WS and Devine SE. An initial map of insertion and deletion (INDEL) variation in the human genome. Genome Res 16: 1182-1190, 2006

34. Kim JI, Ju YS, Park H, Kim S, Lee S, Yi JH, Mudge J, Miller NA, Hong D, Bell CJ, et al: A highly annotated whole-genome sequence of a Korean individual. Nature 460: 1011-1015, 2009

35. Hruban RH, Adsay NV, Albores-Saavedra J, Compton C, Garrett ES, Goodman SN, Kern SE, Klimstra DS, Klöppel G, Longnecker DS, et al: v Am J Surg Pathol 25: 579-586, 2001.

36. Feuk L, Carson AR and Scherer SW: Structural variation in the human genome. Nat Rev Genet 7: 85-97, 2006.

37. Redon R, Ishikawa S, Fitch KR, Feuk L, Perry GH, Andrews TD, Fiegler H, Shapero MH, Carson AR, Chen W, et al: Global variation in copy number in the human genome. Nature 444: 444-454, 2006.

38. Ardito CM, Grüner BM,TakeuchiKK, Lubeseder-Martellato C, Teichmann N, Mazur PK, DelGiorno KE, Carpenter ES, Halbrook CJ, Hall JC, et al: EGF receptor is required for KRAS-induced pancreatic tumorigenesis. Cancer Cell 22: 304-317, 2012.

39. Barton CM, Hall PA, Hughes CM, Gullick WJ and Lemoine NR: Transforming growth factor alpha and epidermal growth factor in human pancreatic cancer. J Pathol 163: 111-116, 1991.

40. Sennino B, Ishiguro-Oonuma T, Wei Y, Naylor RM, Williamson CW, Bhagwandin V, Tabruyn SP, You WK, Chapman HA, Christensen JG, et al: Suppression of tumor invasion and metastasis by concurrent inhibition of c-Met and VEGF signaling in pancreatic neuroendocrine tumors. Cancer Discov 2: 270-287, 2012.

41. Pasche B, Pennison MJ, Jimenez H and Wang M: TGFBR1 and cancer susceptibility. Trans Am Clin Climatol Assoc 125: 300-312, 2014

42. McCarroll SA, Hadnott TN, Perry GH, Sabeti PC, Zody MC Barrett JC, Dallaire S, Gabriel SB, Lee C, Daly MJ, et al: Common deletion polymorphisms in the human genome. Nat Genet 38: 86-92, 2006

43. Nguyen DQ, Webber C and Ponting CP: Bias of selection on human copy-number variants. PLoS Genet 2: e20, 2006. 